\title{
Nocturnal Gastroesophageal Reflux Disease and Microarousals in Obstructive Sleep Apnea
}

\author{
${ }^{1}$ Armaan Mishra, ${ }^{2}$ Arpita Priyadarshini
}

\begin{abstract}
Introduction: Obstructive sleep apnea syndrome (OSAS) is associated with a high frequency of gastroesophageal reflux and arousals. Nocturnal gastroesophageal reflux (nGER) is strongly associated with sleep disturbances and disturbed sleep results in increased nocturnal gastroesophageal reflux.
\end{abstract}

Objectives: It was aimed to study the prevalence of nocturnal gastroesophageal reflux in obstructive sleep apnea (OSA). In our study, we sought to evaluate the correlation between the frequency and type of arousals and episodes of nGER in OSA cases.

Materials and Methods: We conducted an observational study among sixty patients already diagnosed with OSA on the basis of standard sleep criteria. Demographic data, anthropometric measurements, and detailed medical history were recorded. All subjects underwent polysomnography study and filled out the validated Gastroesophagal disease-health related quality of life questionnaire (GERD-HRQL). Statistical analysis was done by the multivariate regression model.

Results: There was a strong significant correlation between apnea-hypopnea index (AHI) and GERD score. ( $r=0.544$, $p=0.002)$. Moreover, respiratory arousal index was also significantly correlated with GERD score $(r=0.370, p=0.040)$. It was found that $\mathrm{AHI}$ was a strong predictor of GERD with a significant regression model $\left(p<0.002, r^{2}=0.0272\right)$. Respiratory arousal index (RAI) was responsible for $13.7 \%\left(r^{2}=0.137\right)$ variance which was statistically significant $p=0.040(p<0.05)$. Desaturation index (DI) also predicted significantly about the gastroesophageal reflux $(p=0.005)$.

Conclusion: The present results suggest that the occurrence of arousals was related to episodes of nGER in OSA.

Funding: This study was part of Short Term Research Studentship (STS) program of the Indian Council of Medical Research (ICMR).

Keywords: Microarousals, nGER, OSAS

How to cite this article: Mishra A, Priyadarshini A. Nocturnal Gastroesophageal Reflux Disease and Microarousals

\footnotetext{
${ }^{1}$ Resident, ${ }^{2}$ Professor and Head

${ }^{1}$ Srirama Chandra Bhanja Medical College and Hospital, Cuttack, India

${ }^{2}$ Department of Physiology, Govt. Medical College, Balangir, Sleep Disorder Clinic and Lab, Srirama Chandra Bhanja Medical College and Hospital, Cuttack, India
}

Corresponding Author: Armaan Mishra, Room no.69, Old Gents Hostel, Srirama Chandra Bhanja Medical College and Hospital, Cuttack, India, e-mail: armaanaamra4u.mishra@ gmail.com in Obstructive Sleep Apnea. Indian Sleep Med 2018;13(2): 21-24.

\section{Source of support: Nil}

Conflict of interest: None

\section{INTRODUCTION}

OSAS is a common disorder characterized by instability of upper airway during sleep resulting in reduction or elimination of airflow. Prevalence of OSA in different socio-economic classes in an urban Indian population has been reported to be $9.3 \% .^{1}$ OSAS is associated with a high frequency of gastroesophageal reflux and arousals. $^{2-4}$

GERD is defined as "a condition that develops when the reflux of stomach contents causes troublesome symptoms and/or complications." ${ }^{\prime 5}$ A recent study has shown a high prevalence of GERD (22\%) in a general population residing in southern India which is much higher than in East Asia. 6

Sleep-related gastroesophageal reflux occurs when gastric contents cross the lower the esophagus sphincter into esophagus and potentially into more proximal sites during sleep time. ${ }^{7}$ Allen et al. ${ }^{8}$ demonstrated that increased acid reflux in the early recumbent period occurs primarily during the recumbent awake and not during the recumbent-asleep periods, suggesting that night-time reflux is associated with difficulty in falling asleep.

The relationship between gastroesophageal reflux and sleep is quite complex. Based on prior research, a gastroesophageal reflux event is temporally associated with an arousal or awakening. ${ }^{9}$

With sleep onset, the upper esophageal pressure decreases and is lowest during N3 sleep, predisposing to aspiration. The upper esophageal sphincter contractile reflex remains intact during REM sleep. Lower esophageal sphincter pressure remains unchanged during sleep. The large negative intrathoracic pressures generated during apnoeic events may draw gastric contents into the oesophagus, particularly in the context of arousals and movements that accompany respiratory instability.

The concept of acid reflux caused by transient lower esophageal sphincter relaxation (TLESR) triggered by arousals $^{10,11}$ remains somewhat controversial. TLESR is the main cause of night-time reflux, but other mechanisms 
such as strain reflux and free reflux are involved in nighttime reflux of patients with GERD. ${ }^{12}$

Although several factors contribute to the association between GERD and sleep disturbances like arousals, nocturnal gastroesophageal reflux is the key factor. Nocturnal gastroesophageal reflux is strongly associated with sleep disturbances. ${ }^{13}$ It includes an increase in the duration of wake stages, a decrease in the duration of NREM sleep, an increase in sleep fragmentation, and frequent stage transitions. ${ }^{14}$ This results in work productivity impairment, poor health-related quality of life and daytime sleepiness. ${ }^{15,16}$

Conversely, disturbed sleep associated with arousals may lead to increased acid reflux. Sleep deprivation appears to cause worsening symptoms of GERD by promoting esophageal mucosal hypersensitivity against gastric acids. ${ }^{17}$ Hence, in our study, we sought to evaluate the correlation between the frequency and type of arousals and episodes of nGER in OSA cases.

\section{MATERIALS AND METHODS}

Study type: Observational study

Study population: Sixty patients already diagnosed with OSA on the basis of standard sleep criteria.

\section{Study Procedures}

The study procedure was approved by the Institutional Review Board. All individuals provided informed consent for participation in the study. Demographic data (age, gender), anthropometric measurements-height, weight, body mass index (BMI) and detailed medical history were recorded. All subjects underwent polysomnography study and filled out the validated GERD-HRQL questionnaire.

\section{Polysomnography}

The diagnostic polysomnograms of sixty patients with OSA are prospectively reviewed. The electroencephalogram (EEG) (C4-A1, C3-A2), left and right electrooculograms (EOG), the submental electromyogram (EMG), oral and nasal flow, a respiratory effort by piezoelectric effort bands, oximetry and right and left anterior tibialis electromyogram are recorded. All studies are recorded NC-PT system and oral flow by the thermostat. Sleep parameters were determined based on American Association of Sleep Medicine (AASM) criteria by a certified polysomnographic technician without knowledge of results of the questionnaire.

Apnea was identified as the cessation of airflow $\geq 10$ s. Obstructive apnea events required the presence of respi- ratory effort throughout the event. $\mathrm{A} \geq 30 \%$ reduction in airflow for at least 10 seconds during sleep accompanied by either a $\geq 3 \%$ desaturation or an arousal was scored as hypopnea. ${ }^{18}$

The AHI was defined as the number of respiratory events (apneas and hypopneas) per hour of total sleep time (TST). OSA was defined as an AHI of at least five events/hour (mild 5-14/h, moderate $15-29 / \mathrm{h}$, and severe $\geq 30 / h$ ).

\section{Scoring of Arousals}

Scoring of arousals is done during all stages of sleep if an abrupt shift of EEG frequency occurs, including alpha, theta or frequencies greater than $16 \mathrm{~Hz}$ (but not spindles), lasting at least three seconds with at least ten seconds of preceding stable sleep. Scoring arousals during REM sleep require an additional increase in submental EMG lasting at least one second. ${ }^{19}$

\section{GERD-Health-Related Quality of Life Questionnaire}

In the Indian setting, a study concluded that 24-h esophageal $\mathrm{pH}$ testing, despite being the gold standard, has no utility in routine clinical settings and hence not considered in this study. ${ }^{20}$ Instead, GERD-HRQL ${ }^{21}$ a self-administered questionnaire in which subjects are asked to report the frequency and severity of their upper gastrointestinal symptoms, is considered. The subjects are assisted by translating the questionnaire into the local language, if needed. The typical features of GERD, i.e., heartburn and regurgitation along with their impact on their daily life are taken into account. The scoring parameters and the corresponding symptom severity assessments made by the physician are associated to ensure proper responsiveness of the questionnaire.

\section{Statistical Analysis}

Data were compiled using Excel software (Microsoft, Redmond, Washington). Data were transferred to IBM statistical package for social sciences (SPSS) windows packaged software for statistical analysis. All variables used in the analyses were checked for skewness. Descriptive statistics were calculated. Numerical variables were summarised with mean \pm standard deviation and categorical variables with frequency and percentage.

Associations between GERD symptoms and OSAS was investigated by calculation of Pearson correlation coefficients using frequency and severity variable from GERD-HRQL questionnaire and calculated indices from polysomnography study. The GERD score was examined in relation to factors that might affect GERD and OSAS including age and BMI. 
Multivariate regression analysis was computed to determine the relationship between GERD score and demographic (age), anthropometric (BMI) and polysomnographic parameters (AHI, arousal indices). A value of $p<0.05$ was considered significant for all statistical analysis.

\section{RESULTS}

\section{Baseline Characteristics}

A total of sixty subjects ranging in age from 27-68 years (mean \pm SD age: $46.9 \pm 9.1$ ) were enrolled in the study. Out of them, 37 (61.7\%) were males, and 23 (38.3\%) were females. Among them, 32 (53.3\%) had a BMI (body mass index) $\geq 30$, considered as obese and $28(46.7 \%)$ of them were overweight BMI: 25-29.9. There were 43 (71.7\%) subjects with severe OSA, 11 (18.3\%) with moderate OSA and 6 (10\%) with mild OSA. All subjects underwent polysomnographic study and filled the GERD-HRQL questionnaire. Summary of patient demographics along with polysomnographic parameters and GERD score is listed in Table 1.

Table 1: Subjects' demographics and polysomnographic parameters $(n=60)$

\begin{tabular}{lcc}
\hline Variable & Mean & $\begin{array}{c}\text { Standard } \\
\text { Deviation }\end{array}$ \\
\hline Age (years) & 46.9 & 9.1 \\
Body mass index $\left(\mathrm{kg} / \mathrm{m}^{2}\right)$ & 32.9 & 5.8 \\
Apnea-hypopnea index & 51.8 & 27.2 \\
Respiratory arousal index & 18.8 & 18.2 \\
Periodic leg movement & & \\
arousal index & 3.7 & 6.5 \\
Snore arousal index & 1.5 & 2.6 \\
Spontaneous arousal index & 11.3 & 11.5 \\
Total arousal index & 36 & 23.1 \\
GERD-HRQL ${ }^{*}$ score & 13.5 & 4.8 \\
\hline
\end{tabular}

*GERD-HRQL-Gastroesophageal disease-health related quality of life

\section{Correlation with GERD Score}

Age and BMI did not correlate with GERD. But, there was a strong significant correlation between AHI and GERD score. $(\mathrm{r}=0.544, \mathrm{p}=0.002)$ Moreover, respiratory arousal index was also significantly correlated with GERD score $(\mathrm{r}=0.370, \mathrm{p}=0.040)$. The GERD score did not significantly correlate with other variables.

\section{Regression}

The relationship between GERD score and other variables was examined to determine factors underlying the development of reflux in OSAS patients. It was found that
AHI was a strong predictor of GERD with a significant regression model. ( $\left.\mathrm{p}<0.002, \mathrm{r}^{2}=0.0272\right)$ Respiratory arousal index was responsible for $13.7 \%\left(r^{2}=0.137\right)$ variance which was statistically significant $p=0.040(p<0.05)$. Desaturation Index also predicted significantly about the gastroesophageal reflux ( $p=0.005)$ (Table 2). Age and BMI along with other polysomnographic parameters fail to predict gastroesophageal reflux significantly.

Table 2: Multivariate linear regression analysis between polysomnographic parameters and gastroesophageal reflux

\begin{tabular}{lccc}
\hline Parameters & Beta & $p$ value & $r$ square \\
\hline AHI & 0.544 & 0.002 & 0.272 \\
RAI & 0.37 & 0.040 & 0.137 \\
DI $^{*}$ & 0.493 & 0.005 & 0.244 \\
\hline
\end{tabular}

AHI-Apnea-hypopnea index; RAI-Respiratory arousal index; DIDesaturation index

*Desaturation index: Desaturations based on 3\% or greater drop from baseline.

$p<0.05$ was considered statistically significant.

\section{DISCUSSION}

It is claimed that gastroesophageal reflux disease especially nGER is common in patients with OSAS. But, prospective studies are limited and contradictory. OSAS and GERD share similar risk factors, and it is unclear whether the co-occurrence of these conditions represents a causal relationship or simply a reflection of co-shared risk factors.

It was shown in the study, how the prevalence of gastroesophageal reflux in OSA is related to the severity of AHI. A significant positive correlation suggests that with an increase in AHI which in turn increases the severity of OSA, the symptom severity of gastroesophageal reflux increases.

Moreover, the correlation between frequency and type of arousals and episodes of nGER is suggested by the significant relation of respiratory arousal index and GERD score.

It is plausible that with increasing severity of OSAS, more GERD would have occurred in a dose-response relationship. The relation between the prevalence of GERD and the factors that served as surrogate indicators of OSAS severity like AHI or nocturnal hypoxemia were examined in several studies. Besides, other indicators of OSAS severity including AHI, oxygen desaturation index, sleep time with oxygen saturation $<90 \%$ did correlate with GERD. These findings suggest that GERD may be increased in OSAS patients.

However, due to the cross-sectional nature of the study, it is difficult to ascertain that they share a causal link or risk factors, but it shows that they are correlated with each other. GERD was significantly associated with 
daytime sleepiness and frequent arousals caused by nocturnal reflux could be an explanation for increased sleepiness. ${ }^{22}$ However, they could not evaluate the arousal index of the patients. Hence, in this study, we analyzed the correlation based on various arousal indices.

Though these studies would have been strengthened by the use of a 'more objective' measure such as the 24-hour $\mathrm{pH}$ probe or upper endoscopy, but lack of data regarding non-acidic reflux and expensiveness limits its purpose. A similar study to determine the direction of any association between GERD and sleep events in patients with OSA, it was found that awakenings and arousals were significantly associated with subsequent onset of a GER event. ${ }^{23}$

In conclusion, it was demonstrated that the prevalence of GERD was significantly increased in subjects with OSAS and the severity of OSAS did influence GERD prevalence. The present results suggest that the occurrence of arousals was related to episodes of nGER in OSA.

\section{REFERENCES}

1. Reddy EV, Kadhivaran T, Mishra HK, Sreenivas V, Handa KK, Sinha S, Sharma SK. Prevalence and risk factors of obstructive sleep apnoea among middle-aged urban Indians: A community based study. Sleep Med 2009 Sep 1;10(8): 913-918.

2. Yang YX, Spencer G, Schutte-Rodin S, Brensinger C, Metz DC. Gastroesophageal reflux and sleep events in obstructive sleep apnea. Eur J Gastroenterol Hepatol 2013 Sep; 25(9):1017-1023.

3. Jung HK, Choung RS, Talley NJ. Gastroesophageal reflux disease and sleep disorders: evidence for a causal link and therapeutic implications. J Neurogastroenterol Motil 2010 Jan; 16(1):22-29.

4. Zanation AM, Senior BA. The relationship between extraesophageal reflux (EER) and obstructive sleep apnea (OSA). Sleep Med Rev 2005 Dec 1; 9(6):453-458.

5. Vakil N, van Zanten SV, Kahrilas P, Dent J, Jones R, Global Consensus Group. The Montreal definition and classification of gastroesophageal reflux disease: A global evidencebased consensus. AmJGastroenterol. 2006 Aug; 101(8):19001920.

6. Hai-Yun Wang, Kondarapassary Balkumaran Leena. Prevalence of gastro-esophageal reflux disease and its risk factors in a community-based population in southern India. BMC Gasteroenterol. 2016 Mar 15; 16(1):36.

7. Bajaj JS, Bajaj S, Dua KS, Joradeh S, Ritmann T, Hofmann C, Sheker R. Influence of sleep stages on upper esophageal sphincter contractile reflex and secondary esophageal peristalsis. Gastroenterology 2006 Jan;130(1):17-25.

8. Allen L, Poh CH, Gasiorowzka A, Malagon I, NavarroRodriguez T, Cui H, Powers J, Moty B, Willis MR, Ashpole $\mathrm{N}$. Increased oeophageal acid exposure at the beginning of the recumbent period is primarily a recumbent-awake phenomenon. Ailment Pharmacol Ther 2010; 32:787794.
9. DiMarino AJ, Banwait KS, Eschinger E, Greenberg A, Dimarino M, Doghramji K, Cohen S. The effect of gastrooesophageal reflux and omeprazole on key sleep parameters. Aliment Pharmacol Ther 2005; 22:325-329.

10. Dent J, Dodds WJ, Friedman RH, Sekiguchi T, Hogan WJ, Andorfer RC, Petrie DJ. Mechanism of gastroesophageal reflux in recumbent asymptomatic human subjects. J Clin Invest 1980 Feb 1; 65(2): 256-267.

11. Schneider JH, Küper MA, Königsrainer A, Brücher BL. Transient lower esophageal sphincter relaxation and esophageal motor response. J Surg Res. 2010 Apr; 159(2):714-719.

12. Kuribayashi S1, Kusano M, Kawamura O, Shimoyama $Y$, Maeda M, Hisada T, Ishizuka T, Dobashi K, Mori M. Mechanism of gastroesophageal reflux in patients with obstructive sleep apnea syndrome. Neurogastroenterol Motil 2010 Jun; 22 (6): 611-e172.

13. Fujiwara $Y$, Arakawa T, Fass R. Gastroesophageal reflux disease and sleep disturbances. J Gasroenterol 2012 Jul 1; 47(7):760-769.

14. Nakahara K, Fujiwara $Y$, Tsukahara T, Yamagami H, Tanigawa T, Shiba M, Tominaga K, Watanabe T, Urade Y, Arakawa T. Acid reflux directly causes sleep disturbances in rat with chronic esophagitis. PloS one. 2014 Sep 12;9(9):e106969.

15. Mody R, Bolge SC, Kannan H, Fass R. Effects of gastroesophageal reflux disease on sleep and outcomes. Clin Gastroenterol Hepatol 2009 Sep 1; 7(9): 953-959.

16. Dubois RW, Aguilar D, Fass R, Orr WC, Elfant AB, Dean BB, Harper AS, YU HT, Melmed GY, Lynn R, Singh A. Consequences of frequent nocturnal gastro oesophageal reflux disease among employed adults: symptom severity, quality of life and work productivity. Alimentary pharmacology \& therapeutics. 2007 Feb;25(4):487-500.

17. Schey R, Dickman R, Parthasarathy S, Quan SF, Wendel C, Merchant J, Powers J, Han B, Van Handel D, Fass R. Sleep deprivation is hyperalgesic in patients with gastroesophageal reflux disease. Gastroenterology. 2007 Dec 1;133(6):1787-95.

18. American Academy of Sleep Medicine International classification of sleep disorders, 3rd edition: Diagnostic and coding manual. Westchester, Illinois: American Academy of Sleep Medicine 2014.

19. Iber, C.; Ancoli-Israel, S.; Chesson, AL., et al. The AASM Manual for the Scoring of Sleep and Associated Events. American Academy of Sleep Medicine; West Chester, IL: 2007.

20. Madan K, Ahuja V, Gupta DS, Sharma MP, Bal C, Kapoor A. Impact of 24-hr oesophageal $\mathrm{pH}$ monitoring on the diagnosis of gastroesophageal reflux disease: defining the gold standard. J Gastroenterol Hepatol. 2005; 20:30-37.

21. Velanovich V. The development of the GERD-HRQL symptom severity instrument. Dis Esophagus 2007 Apr 1; 20(2):130-134.

22. Ozen K. Basoglu, Rukiye Vardar, Serhat bor Tazbakan MS, Ucar ZZ, Ayik S, Kose T. Obstructive sleep apnea syndrome and gastroesophageal reflux disease: the importance of obesity and gender. Sleep and Breathing, 2015, 19(2):585-592.

23. Yu Xiao Yang, Spencer G, Schulte-Rodin S, Brensinger C, Mtz TC. Gatroesophageal reflux and sleep events in OSA; European Journal of gasteroenerology and hepatology, 2013;25:1017-1023. 